\title{
Pacific
}

Journal of

Mathematics

\section{ON METRICS DEFINED BY MODULES}

JAMES ALLISTER JeNKINS 


\title{
ON METRICS DEFINED BY MODULES
}

\author{
JAMES A. JENKINS
}

\begin{abstract}
Jacqueline Ferrand gave a very general definition of a conformal invariant $\lambda_{G}(x, y)$ for a domain $G$ by the use of modules of curve families and showed that, in dimension $n, \lambda_{G}(x, y)^{-1 / n}$ is a metric. The question as to whether $\lambda_{G}(x, y)^{-1 /(n-1)}$ is itself a metric was raised by Vuorinen and studied by him and Jacqueline Ferrand. In particular he asked whether this held for $n=2$ and $G$ the punctured plane. In this paper it is shown that the answer is affirmative for any domain of finite connectivity on the sphere.
\end{abstract}

1. Jacqueline Ferrand [4] gave a very general method for defining metrics by the use of modules of curve families. Let $G$ be a domain in $R^{n}, x, y \in G, C_{x}, C_{y}$ connected closed subsets of $G$ with $x \in$ $C_{x}, y \in C_{y}, \mathrm{Cl} C_{x} \cap \partial G \neq 0, \mathrm{Cl} C_{y} \cap \partial G \neq 0$. Let $\Delta\left(C_{x}, C_{y}, G\right)$ denote the family of all curves in $G$ joining $C_{x}$ and $C_{y}, M()$ denote the module of a curve family. Let

$$
\lambda_{G}(x, y)=\text { g.l.b. } M\left(\Delta\left(C_{x}, C_{y}, G\right)\right)
$$

taken over the above configurations. She proved by a standard extremal metric argument that $\lambda_{G}(x, y)^{-1 / n}$ is a metric on $G$. The question has been raised by her and especially by Vuorinen (see [8], p.193) under what circumstances $\lambda_{G}(x, y)^{-1 /(n-1)}$ is itself a metric. In [2] it is shown that when $G$ is the $n$-ball $B^{n}=\left\{x \in R^{n} ;\|x\|<\right.$ $1\}, \lambda_{G}(x, y)^{-p}$ is a metric if and only if $p \in[0,1 /(n-1)]$. In the summer of 1987 Vuorinen raised the above question to me, asking whether it held even in the case of the punctured plane. Sometime after that I gave him a very simple proof answering that question in the affirmative. In the present paper it will be shown that the same is true for any domain of finite connectivity on the sphere.

2. We begin by treating the special case mentioned above in which the essence of the proof is revealed without involving some of the technical matters which occur in the general case. 
THEOREM 1. Let $G$ be the domain obtained by deleting from the sphere two points which we may take to be 0 and $\infty$. Let $x, y \in G$. Let $C_{x}$ be a continuum containing $x$ and 0 or $\infty$ and $C_{y}$ a continuum containing $y$ and the other. Let $M\left(C_{x}, C_{y}\right)$ denote the module of the family of curves joining $C_{x}, C_{y}$. Let $\lambda_{G}(x, y)$ be the g.l.b. of this quantity for all such configurations. Then $\lambda_{G}(x, y)^{-1}$ is a metric on $G$.

Proof. Evidently we may assume $C_{x}, C_{y}$ disjoint. Then they determine a doubly-connected domain $D(x, y)$ and $M\left(C_{x}, C_{y}\right)$ is the reciprocal of the module $M(x, y)$ of $D(x, y)$. The level curves of this domain determine a free (unsensed) homotopy class $\mathcal{H}$ in $G-\{x, y\}$. The module of this homotopy class is equal to the maximal module of a domain such as $D(x, y)$. This follows from the present author's fundamental theorem [5]. There is a homotopy class $\mathcal{H}^{*}$ (unique except in certain special cases) for which the module is maximal. We call this value $m(x, y)$; it is evidently equal to $\lambda_{G}(x, y)^{-1}$. For definiteness we will assume that it arises from continua joining $x$ and $0, y$ and $\infty$.

Let now $z \in G$ be distinct from $x$ and $y$. $\mathcal{H}^{*}$ will determine homotopy classes $\Gamma_{1}, \Gamma_{2}$ of Jordan curves separating 0 and $x$ from $z$ and $\infty$ and separating 0 and $z$ from $y$ and $\infty$. Let $\rho_{1}(w)|d w|, \rho_{2}(w)|d w|$ be the extremal metrics for these classes. Let $\rho=\max \left(\rho_{1}, \rho_{2}\right)$. Then $\rho(w)|d w|$ is an admissible metric for the family

$$
\mathcal{H}^{*}-\{\text { curves through } z\} \text {. }
$$

Since the latter set of curves has module zero we have

$$
m(x, y) \leq M\left(\Gamma_{1}\right)+M\left(\Gamma_{2}\right) \leq m(x, z)+m(z, y)
$$

This completes the proof of Theorem 1 .

3. THEOREM 2. Let $G$ be a domain of finite connectivity on the sphere. Let $x, y \in G$. Let $C_{x}$ be a (relatively) closed subset of $G$ with $x \in C_{x}$ and $C l C_{x} \cap \partial G \neq 0, C_{y}$ a similar subset of $G$ with $y \in C_{y}$ and $\mathrm{ClC}_{y} \cap \partial G \neq 0$. Let $\Delta\left(C_{x}, C_{y}, G\right)$ denote the family of all curves in $G$ joining $C_{x}$ and $C_{y}, M\left(\Delta\left(C_{x}, C_{y}, G\right)\right)$ its module. Set

$$
\lambda_{G}(x, y)=\text { g.l.b. } M\left(\Delta\left(C_{x}, C_{y}, G\right)\right)
$$


taken over the above configurations. Then $\lambda_{G}(x, y)^{-1}$ is a metric on $G$.

Proof. Evidently we may assume $C_{x}, C_{y}$ disjoint. Then the extremal metric for the module problem defining $M\left(\Delta\left(C_{x}, C_{y}, G\right)\right)$ is given by $|\operatorname{grad} u||d z|$ where $u$ is a bounded harmonic function on $G$ with boundary values 0 on $C_{x}, 1$ on $C_{y}$ and vanishing normal derivative on the remaining hyperbolic boundary of $G$ in terms of border uniformizers (see [1], p.65, where a highly detailed discussion is given or [3], p.367). Each level set of $u$ is made up of a Jordan curve or a finite number of arcs joining boundary components of $G$. The module of the family of level sets is $M\left(\Delta\left(C_{x}, C_{y}, G\right)\right)^{-1}$. The level sets determine a family of homotopy classes of corresponding Jordan curves or arcs (for the latter this being understood in the sense indicated in [5]). Let $\mathrm{Cl} C_{x}$ meet the boundary component $A_{x}$ of $G, \mathrm{Cl} C_{y}$ the boundary component $A_{y}$. If either is a point boundary they must be disjoint, otherwise they can coincide. Let $\Gamma(x, y)$ denote the family of elements each represented by a selection from homotopy classes possessing the same separating properties as above. It is clear that

$$
M\left(\Delta\left(C_{x}, C_{y}, G\right)\right)^{-1} \leq M(\Gamma(x, y)) .
$$

The extremal metric for the module problem determining $M(\Gamma(x, y))$ is given by $|Q(z)|^{1 / 2}|d z|$ where $Q(z) d z^{2}$ is a quadratic differential on $G$ negative on hyperbolic border components of $G$. If $A_{x}$ or $A_{y}$ is a point boundary, $Q(z) d z^{2}$ will have a simple pole there and it will have a trajectory arc with end points at $x$ and $A_{x}$ or at $y$ and $A_{y}$ as the case may be. If $A_{x}$ or $A_{y}$ is non-degenerate there will be a trajectory arc of $Q(z) d z^{2}$ tending from $x$ or $y$ to a border element $P_{x}$ or $P_{y}$ of $A_{x}$ or $A_{y}$. These play the role of $C_{x}$ or $C_{y}$. These results require a slight extension of the results of [5] with quadrangles possibly being replaced by families of quadrangles using the definition of module found in [6]. They are readily established by the methods given in [7]. Thus $\lambda_{G}(x, y)^{-1}$ is seen to be the maximum of $M(\Gamma(x, y))$ attained for a family of homotopy classes with elements $\Gamma$ separating $x$ and a point on a suitable $A_{x}$ from $y$ and a point on a suitable $A_{y}$. If $z$ is a point of $G$ distinct from $x$ and $y$ and $\Gamma_{1}, \Gamma_{2}$ are the subsets of $\Gamma$ separating $x$ from $z$ and $y$ or $x$ and $z$ from $y$ as in the proof of Theorem 1 we have 


$$
\lambda_{G}(x, y)^{-1}=m(\Gamma) \leq m\left(\Gamma_{1}\right)+m\left(\Gamma_{2}\right) \leq \lambda_{G}(x, z)^{-1}+\lambda_{G}(z, y)^{-1} .
$$

This completes the proof of Theorem 2 .

\section{REFERENCES}

[1] L. Ahlfors, Conformal Invariants, McGraw-Hill, New York, 1973.

[2] G. Anderson, M. Vamanamurthy and M. Vuorinen, Special functions of quasiconformal theory, Exposition. Math., 7 (1989), 97-136.

[3] A. Beurling, Collected Works, vol. 1, Birkhäuser, Boston, Basel, Berlin 1989.

[4] J. Ferrand, Invariants conformes globaux sur les variétés Riemanniennes, J. Differential Geom., 8 (1973), 487-510.

[5] James A. Jenkins, On the existence of certain general extremal metrics, Annals of Math., 66 (1957), 440-453.

[6] - Some uniqueness results in the theory of symmetrization II, Annals of Math., 75 (1962), 223-230.

[7] - On the existence of certain general extremal metrics II, to appear.

[8] M. Vuorinen, Conformal geometry and quasiregular mappings, Lecture Notes in Mathematics 1319, Springer-Verlag, Berlin, Heidelberg 1988.

Received July 21, 1992.

WASHINGTON UNIVERSITY

St. Louis, MO 63130-4899 



\section{PACIFIC JOURNAL OF MATHEMATICS}

Volume $167 \quad$ No. $2 \quad$ February 1995

Existence of shortest directed networks in $\mathbb{R}^{2}$

201

MANUEL AlFARO GARCIA

Hecke characters of singular Drinfel'd modules

215

SUNGHAN BAE

Factorization method for a bimeromorphic morphism

231

Jose PEREZ BLANCO

$L^{p}$ estimates for operators associated to flat curves without the Fourier 243 transform

Anthony Carbery, James Thomas Vance, JR., Stephen

WAINGER, DAVID K. WATSON and JAMES WRIGHT

$S$-integer points on elliptic curves

263

ROBERT HOWARD GROSS and JOSEPH SILVERMAN

On metrics defined by modules

JAMES ALLISTER JENKINS

Conditional Wiener integrals. II

ChUll PARK and DAVID LeE SKOUG

On a Plancherel formula for certain discrete, finitely generated, torsion-free nilpotent groups

CAROLYN PFEFFER JOHNSTON

Desingularizations of some unstable orbit closures

MARK STEPHEN REEDER

Determining multiplicities of half-integral weight newforms

Thomas Richard SHEMANSKE and LyNne WALLING

Generation of integral orthogonal groups over dyadic local fields 